Track: Financial Markets, Investments and Risk Management

William Eid Junior

Full Professor

FGV/EAESP

Escola de Administração de Empresas de São Paulo

Fundação Getúlio Vargas

Av. Nove de Julho $2029 \quad 10^{\circ}$ andar

São Paulo Brasil CEP 01313-902

Phone: $55113281-7899$

Fax: $55113284-1789$

weid@fgvsp.br

Indexing: Eid Jr, William

Ricardo Ratner Rochman

Professor

FGV/EAESP

Escola de Administração de Empresas de São Paulo

Fundação Getúlio Vargas

Av. Nove de Julho $2029 \quad 10^{\circ}$ andar

São Paulo Brasil CEP 01313-902

Phone: 5511 3281-7899

Fax: $55113284-1789$

rrochman@fgvsp.br

Indexing: Rochman, Ricardo Ratner

Key words

Mutual funds, Brazil, Active and Passive Management 


\title{
Does active management add value ? The Brazilian mutual fund market
}

\author{
$\underline{\text { Abstract }}$ \\ Does active management add or destroy value ? With a sample of 699 with four different main categories: stocks, fixed \\ income, hedge and exchange rate mutual funds we conclude that the active management add value to investors in stocks and \\ hedge funds. But in fixed income mutual funds the evidence is against the active management. We also analyze the \\ determinants of significant alphas. For stocks and hedge funds the evidence suggests that old, big and active funds generate \\ biggest alphas. In fixed income funds the evidence is not clear, only a positive relationship between size and alphas could be \\ found.
}

\section{$\underline{\text { 1. Introduction }}$}

From an investor point of view there is a fundamental question: is it better to invest in a passive fund or in an active one? In other words, do the active managers have superior skills to select better investments?

Passive management means having a portfolio that mimics the market, that is, a portfolio that have all the available assets in the same proportion as they exists in the market. The main advantages of this king of management are:

a. Passive funds have slow operational costs.

b. As the investment game is a zero sum game and the active funds have great operational costs, they must show a worse performance then the passive ones.

c. In efficient markets there is no reason for the existence of active funds better performance.

An active portfolio own different assets than the market portfolio. Managers of this kind of fund seek for better performance than the market. The main arguments in favor of active management are:

a. Markets are not perfectly efficient

b. In bear markets active managers add value.

c. Hot hands effect exists. Funds with better past performance tend to have better performance in the future. This effect clearly shows the advantage of active management.

Our study tries to answer the original question in the Brazilian mutual fund industry.

\section{$\underline{\text { 2. Relevant literature }}$}


There are a lot of academic studies looking for the difference between active and passive management. Jensen (1968) is the seminal study. He analyses the performance of 115 mutual funds during the 20 years between 1945 e 1954 . The main conclusion is against the value of active management. He shows that in average the funds were not able to beat a passive strategy. More than this, the funds were not capable to cover the operational expenses. The analysis was based on the today well know Jensen's alpha.

More recently, Grinblat and Titman (1989) shows that the smallest aggressive growth funds, and growth funds have a positive gross performance when compared to passive funds. But they also have the biggest operational expenses resulting in a non abnormal liquid performance. They also use the Jensen's alpha.

Cahart (1997) uses a factor model that includes operational expenses that almost completely explain the persistence phenomenon in mutual funds and concludes that there is no evidence of superior performance with active management.

Chen, Jegadeesh and Wermers (2000) analyses the portfolio assets in the mutual funds. Their sample covers all the mutual funds in USA during the period comprised between 1975 and 1995. They show that the stocks that are in the mutual funds portfolios do not show better performance than the others. But at the same time, looking for the mutual fund trades, they discovered that the most actively traded stocks shows better performance than the others. But this advantage has short life, less than one year. And the funds usually stay with stocks more than one year avoiding transaction costs.

Kallberg, Liu and Trzcinka (2000) analyses the performance of 128 REITs - Real State Investment Trusts between 1986 and 1998. They concluded that REITs active managers add value. These funds have positive Jensen's alpha, even when considering the performance liquid of operational expenses. And the active ones show biggest Alphas than the passive ones. Another interesting conclusion is that the managers add value when the market shows a poor performance.

Wermers (2000) is another work that supports the hypothesis of active management value. He studies the period between 1974 and 1995 looking for the different components of funds performance as expenses, turnover, liquid return and others. He shows that the funds had a superior gross return of $1,3 \%$, comparing to the market, and that $0,6 \%$ of the $1,3 \%$ comes from stocks' specific characteristics and the other $0,7 \%$ from the superior skills in selecting stocks. But the liquid return is $1 \%$ bellow the market, mainly due to operational expenses and poor performance of other assets in the portfolios than stocks.

Lhabitant (2001) concludes that in the Swiss mutual fund market the active management does not add value to the investor. He analyses the performance of 60 mutual funds between 1977 and 1990.

Kacperczyk, Sialm and Zheng (2005) studying 1711 mutual funds in the USA between 1984 and 1999 and using a dual database - mutual funds returns and portfolio composition - concludes that active management adds value. Mainly in funds that are heavily concentrated in one industry. 


\section{Data and Methodology}

The Brazilian mutual fund industry has in the beginning of 2006 aproximately 4000 funds. But only 1200 are open to the public, the other ones being exclusives or closed to the public. This 1200 were our main target. From these 1200 mutual funds we excluded the ones without 5 years of live and from the remaining we selected the ones that are classified in the Anbid (National Bank Association) as Stock Funds, Exchange Rate Funds, Fixed Income Funds and Hedge Funds.

Our sample contains 699 different mutual funds between 01/31/2001 and 01/31/2006. The total NAV is R\$ R\$ 365.360.633.960,84 and accounts for 46,17\% from the total industry NAV. Table I shows the number of funds in each category and also their nature in terms of management according to the Anbid classification.

Table I: The sample

\begin{tabular}{|l|r|r|r|}
\hline Number of Funds & \multicolumn{1}{|c|}{ Active } & Passive & \multicolumn{1}{c|}{ Total } \\
\hline Stock & 129 & 34 & 163 \\
\hline Exchange Rate & 15 & 40 & 55 \\
\hline Hedge & 118 & 21 & 139 \\
\hline Fixed Income & 148 & 194 & 342 \\
\hline Total & $\mathbf{4 1 0}$ & $\mathbf{2 8 9}$ & $\mathbf{6 9 9}$ \\
\hline
\end{tabular}

As a proxy to the risk free rate we used three different rates: the CDI (Interbank Depositary Receipts), the Selic (Treasury Bonds Rate) and Savings Account Rate (poupança). We collected these rates in the Economatica database.

For each fund category we used four different market portfolios. Two directly associated with the category benchmark, another one associated with the category average monthly log-returns and the last one associated with of our sample average monthly log-return. Table 2 shows the different combinations of risk-free rate and market portfolio for each mutual fund category:

Table II: Market and Risk-Free Rate

\begin{tabular}{|c|c|c|c|c|c|c|c|}
\hline \multicolumn{8}{|c|}{ Category } \\
\hline \multicolumn{2}{|c|}{ Stock } & \multicolumn{2}{|c|}{ Exchange Rate } & \multicolumn{2}{|c|}{ Hedge } & \multicolumn{2}{|c|}{ Fixed Income } \\
\hline Market & $\begin{array}{c}\text { Risk-Free } \\
\text { Rate }\end{array}$ & Market & $\begin{array}{c}\text { Risk-Free } \\
\text { Rate }\end{array}$ & Market & $\begin{array}{c}\text { Risk-Free } \\
\text { Rate }\end{array}$ & Market & $\begin{array}{c}\text { Risk-Free } \\
\text { Rate }\end{array}$ \\
\hline IBOVESPA & CDI & Dólar (PTAX) & CDI & IBOVESPA & CDI & SELIC & CDI \\
\hline IBOVESPA & Savings & Dólar (PTAX) & Savings & IBOVESPA & Savings & SELIC & Savings \\
\hline IBOVESPA & SELIC & Dólar (PTAX) & SELIC & IBOVESPA & SELIC & & \\
\hline IBrX & CDI & Euro & CDI & SELIC & CDI & & \\
\hline IBrX & Savings & Euro & Savings & SELIC & Savings & CDI & Savings \\
\hline $\mathrm{IBrX}$ & SELIC & Euro & SELIC & & & & \\
\hline Category Average & CDI & Category Average & CDI & Category Average & CDI & Category Average & CDI \\
\hline Category Average & Savings & Category Average & Savings & Category Average & Savings & Category Average & Savings \\
\hline Category Average & SELIC & Category Average & SELIC & Category Average & SELIC & Category Average & SELIC \\
\hline General Average & $\mathrm{CDI}$ & General Average & CDI & General Average & CDI & General Average & CDI \\
\hline General Average & Savings & General Average & Savings & General Average & Savings & General Average & Savings \\
\hline General Average & SELIC & General Average & SELIC & General Average & SELIC & General Average & SELIC \\
\hline
\end{tabular}


Data for Ibovespa Index, IBX Index and USD/R \$ exchange rate were collected from the Economatica database. The Euro/R \$ exchange rate was collected from the Central Bank database. The daily return and mutual funds NAV were collected from the Anbid database.

Our null hypothesis is that the active managed fund performance is not superior to the passive managed fund performance. To test this hypothesis we used the Jensen's (1968) model that measures the mutual fund performance trough the following regression, using the MSE method:

$$
R_{P t}-R_{f t}=\alpha_{P}+\beta_{P}\left(R_{M t}-R_{f t}\right)+\varepsilon_{P t}
$$

Were $\mathrm{R}_{\mathrm{Pt}}$ is the log-return in month $\mathrm{t}$ from the mutual fund $\mathrm{P}, \mathrm{R}_{\mathrm{ft}}$ is one of our risk-free rates, $\mathrm{R}_{\mathrm{Mt}}$ is the log-return of one of our market portfolios, $\alpha_{\mathrm{P}}$ measures the ability in selecting assets, $\beta_{\mathrm{P}}$ measures the sensibility between the mutual fund logreturn and the market portfolio log-return and $\varepsilon_{\mathrm{Pt}}$ is a normally distributed variable with mean $=0$ and independent of $R_{\mathrm{Pt}}$. $\alpha_{p}$ can be seen as the return difference between two portfolios: the one that we are studying and a passive one were with $\beta_{P}$ units invested in the market portfolio and $1-\beta_{\mathrm{P}}$ units invested in the risk-free rate. A positive $\alpha_{\mathrm{p}}$ shows superior skills in micro-previsions, and a negative one shows poor ability or much operational expenses.

In estimating the above equation, even with iid returns, we can find conditional heterodasticity that compromises the MSE estimation efficiency. Henriksson (1984), and Chang e Lewellwn (1984) concludes that the errors are minimum, but we decide to avoid this error correcting to heterocedasticity using the White (1980) procedure getting t-statistics adjusted and with asymptotic validity.

We also look for the determinants of the mutual fund returns. Do to this we used cross-section regressions with the MSE method. We regress the Jensen's alpha of each fund against the following variables:

a. Active or passive management $\left(\right.$ Active $\left._{P}\right)$ : a dummy variable that are 1 if the management is active and 0 otherwise. We use the mutual funds that show a significant alpha, positive or negative. We expected a positive and significant result for this variable.

b. Mutual fund Size $\left(\operatorname{Ln}\left(N A V_{P}\right)\right)$ : we use the log of NAV for each fund in $01 / 31 / 2006$. Economic reasons like scale and bargain power can justify the superior performance of greatest funds. But flexibility can justify a superior performance of smallest funds.

c. National (National $\mathrm{P}$ ): a dummy variable with is 1 if the mutual fund is managed by a Brazilian financial organization and 0 if we are dealing with a foreign organization. We expected that the Brazilian managers do better than foreign due to flexibility, market knowledge or access to private information. 
d. Mutual fund age $\left(\operatorname{Ln}\left(\operatorname{Age}_{P}\right)\right.$ : the number of days log between the first day of mutual fund existence and 01/31/2006. Younger mutual funds usually have biggest operational expenses like marketing. We expected that older mutual funds have a better performance than the youngest ones.

e. Standard deviation $\left(\sigma_{\left(R_{P_{t}}-R_{f t}\right)}\right)$ : the standard deviation of the difference between the mutual fund return and the risk-free rate was used as a proxy for mutual fund risk. The greatest the Jensen's Alpha, the greatest the risk. That's what we expected.

Only mutual funds with Jensen's Alpha significant at $15 \%$ level were considered for the cross section regression. For each fund category we run the following regression:

$$
\begin{aligned}
& \alpha_{P}=\beta_{0}+\beta_{1} \cdot \text { Active }_{P}+\beta_{2} \cdot \operatorname{Ln}\left(N A V_{P}\right)+\beta_{3} \cdot \text { National }_{P}+\beta_{4} \cdot \operatorname{Ln}\left(\text { Age }_{P}\right)+\varepsilon_{P} \\
& \alpha_{P}=\beta_{0}+\beta_{1} \cdot \text { Active }_{P}+\beta_{2} \cdot \operatorname{Ln}\left(N A V_{P}\right)+\beta_{3} \cdot \text { National }_{P}+\beta_{4} \cdot \operatorname{Ln}\left(\text { Age }_{P}\right)+\beta_{5} \cdot \sigma_{\left(R_{P_{t}}-R_{f t}\right)}+\varepsilon_{P}
\end{aligned}
$$

The difference between the two regressions above is the standard deviation insertion in the second one.

\section{Results}

The first question that we want to answer is: does the active management of mutual funds add or destroy value? That is, do we found significant Jensen's Alphas? The answer depends on the mutual fund category. The following tables present the results by category.

\begin{tabular}{|c|c|c|c|c|c|c|c|c|}
\hline \multicolumn{9}{|c|}{ Fund Category: Stocks } \\
\hline \multirow[b]{2}{*}{ Market } & \multirow[b]{2}{*}{$\begin{array}{c}\text { Risk-Free } \\
\text { Rate }\end{array}$} & \multicolumn{2}{|c|}{ Number of Active Funds } & \multicolumn{2}{|c|}{ Number of Passive Funds } & \multicolumn{3}{|c|}{ Total } \\
\hline & & $\begin{array}{c}\text { With } \\
\text { Significant } \\
\text { Alpha }\end{array}$ & $\begin{array}{c}\text { Without } \\
\text { Significant } \\
\text { Alpha }\end{array}$ & $\begin{array}{c}\text { With } \\
\text { Significant } \\
\text { Alpha }\end{array}$ & $\begin{array}{c}\text { Without } \\
\text { Significant } \\
\text { Alpha }\end{array}$ & $\begin{array}{c}\text { With } \\
\text { Significant } \\
\text { Alpha }\end{array}$ & $\begin{array}{c}\text { Without } \\
\text { Significant } \\
\text { Alpha }\end{array}$ & General \\
\hline IBOVESPA & CDI & 63 & 66 & 20 & 14 & 83 & 80 & 163 \\
\hline IBOVESPA & Savings & 66 & 63 & 20 & 14 & 86 & 77 & 163 \\
\hline IBOVESPA & SELIC & 63 & 66 & 20 & 14 & 83 & 80 & 163 \\
\hline IBrX & CDI & 73 & 56 & 30 & 4 & 103 & 60 & 163 \\
\hline IBrX & Savings & 73 & 56 & 30 & 4 & 103 & 60 & 163 \\
\hline IBrX & SELIC & 73 & 56 & 30 & 4 & 103 & 60 & 163 \\
\hline Category Average & CDI & 72 & 57 & 27 & 7 & 99 & 64 & 163 \\
\hline Category Average & Savings & 78 & 51 & 28 & 6 & 106 & 57 & 163 \\
\hline Category Average & SELIC & 72 & 57 & 27 & 7 & 99 & 64 & 163 \\
\hline General Average & CDI & 120 & 9 & 25 & 9 & 145 & 18 & 163 \\
\hline General Average & Savings & 126 & 3 & 34 & 0 & 160 & 3 & 163 \\
\hline General Average & SELIC & 121 & 8 & 28 & 6 & 149 & 14 & 163 \\
\hline
\end{tabular}

TABLE III: Stock Funds and Alphas

Table III shows the results for stocks mutual funds. The result depends on the mutual fund benchmark. But only half of the mutual funds show significant alphas. This evidence indicates the existence of value aggregation or destruction. 
TABLE IV: Exchange Rate Funds and Alphas

\begin{tabular}{|c|c|c|c|c|c|c|c|c|}
\hline \multicolumn{9}{|c|}{ Fund Category: Exchange Rate } \\
\hline \multirow[b]{2}{*}{ Market } & \multirow[b]{2}{*}{$\begin{array}{c}\text { Risk-Free } \\
\text { Rate }\end{array}$} & \multicolumn{2}{|c|}{ Number of Active Funds } & \multicolumn{2}{|c|}{ Number of Passive Funds } & \multicolumn{3}{|c|}{ Total } \\
\hline & & $\begin{array}{c}\text { With } \\
\text { Significant } \\
\text { Alpha }\end{array}$ & $\begin{array}{c}\text { Without } \\
\text { Significant } \\
\text { Alpha }\end{array}$ & $\begin{array}{c}\text { With } \\
\text { Significant } \\
\text { Alpha }\end{array}$ & $\begin{array}{c}\text { Without } \\
\text { Significant } \\
\text { Alpha }\end{array}$ & $\begin{array}{c}\text { With } \\
\text { Significant } \\
\text { Alpha }\end{array}$ & $\begin{array}{c}\text { Without } \\
\text { Significant } \\
\text { Alpha }\end{array}$ & General \\
\hline \begin{tabular}{|l} 
Dólar (PTAX) \\
\end{tabular} & CDI & 1 & 14 & 2 & 38 & 3 & 52 & 55 \\
\hline Dólar (PTAX) & Savings & 2 & 13 & 4 & 36 & 6 & 49 & 55 \\
\hline Dólar (PTAX) & SELIC & 1 & 14 & 2 & 38 & 3 & 52 & 55 \\
\hline Euro & CDI & 1 & 14 & 2 & 38 & 3 & 52 & 55 \\
\hline Euro & Savings & 1 & 14 & 1 & 39 & 2 & 53 & 55 \\
\hline Euro & SELIC & 1 & 14 & 2 & 38 & 3 & 52 & 55 \\
\hline Category Average & CDI & 1 & 14 & 15 & 25 & 16 & 39 & 55 \\
\hline Category Average & Savings & 3 & 12 & 15 & 25 & 18 & 37 & 55 \\
\hline Category Average & SELIC & 1 & 14 & 15 & 25 & 16 & 39 & 55 \\
\hline General Average & CDI & 12 & 3 & 38 & 2 & 50 & 5 & 55 \\
\hline General Average & Savings & 6 & 9 & 40 & 0 & 46 & 9 & 55 \\
\hline General Average & SELIC & 12 & 3 & 38 & 2 & 50 & 5 & 55 \\
\hline
\end{tabular}

Exchange rate mutual funds results are in table IV. And here we do not have the same evidence that we found in stocks mutual funds. The only benchmark where we found significant alphas was in general average. And by construction this benchmark must show worse performance, what conducts to diverse results.

TABLE V: Hedge Funds and Alphas

\begin{tabular}{|c|c|c|c|c|c|c|c|c|}
\hline \multicolumn{9}{|c|}{ Fund Category: Hedge } \\
\hline \multirow[b]{2}{*}{ Market } & \multirow[b]{2}{*}{$\begin{array}{c}\text { Risk-Free } \\
\text { Rate }\end{array}$} & \multicolumn{2}{|c|}{ Number of Active Funds } & \multicolumn{2}{|c|}{ Number of Passive Funds } & \multicolumn{3}{|c|}{ Total } \\
\hline & & $\begin{array}{c}\text { With } \\
\text { Significant } \\
\text { Alpha }\end{array}$ & $\begin{array}{c}\text { Without } \\
\text { Significant } \\
\text { Alpha }\end{array}$ & $\begin{array}{c}\text { With } \\
\text { Significant } \\
\text { Alpha }\end{array}$ & $\begin{array}{c}\text { Without } \\
\text { Significant } \\
\text { Alpha }\end{array}$ & $\begin{array}{c}\text { With } \\
\text { Significant } \\
\text { Alpha }\end{array}$ & $\begin{array}{c}\text { Without } \\
\text { Significant } \\
\text { Alpha }\end{array}$ & General \\
\hline IBOVESPA & CDI & 0 & 118 & 3 & 18 & 3 & 136 & 139 \\
\hline IBOVESPA & Savings & 2 & 116 & 5 & 16 & 7 & 132 & 139 \\
\hline IBOVESPA & SELIC & 0 & 118 & 3 & 18 & 3 & 136 & 139 \\
\hline \begin{tabular}{|l|} 
SELIC \\
\end{tabular} & CDI & 117 & 1 & 21 & 0 & 138 & 1 & 139 \\
\hline \begin{tabular}{|l|} 
SELIC \\
\end{tabular} & Savings & 90 & 28 & 19 & 2 & 109 & 30 & 139 \\
\hline \begin{tabular}{|l} 
Category Average \\
\end{tabular} & CDI & 46 & 72 & 18 & 3 & 64 & 75 & 139 \\
\hline Category Average & Savings & 108 & 10 & 20 & 1 & 128 & 11 & 139 \\
\hline Category Average & SELIC & 44 & 74 & 18 & 3 & 62 & 77 & 139 \\
\hline General Average & CDI & 14 & 104 & 8 & 13 & 22 & 117 & 139 \\
\hline General Average & Savings & 112 & 6 & 18 & 3 & 130 & 9 & 139 \\
\hline General Average & SELIC & 15 & 103 & 9 & 12 & 24 & 115 & 139 \\
\hline
\end{tabular}

Table V presents the results for hedge funds with mixed evidence. As in stocks mutual funds, the results depend on the used

benchmark. The results are consistent with the use of CDI by the majority of mutual fund managers. In the case of Ibovespa as benchmark, we cannot find a great number of significant alphas. As expected as the managers do not use this benchmark. In the case of category average and general average we found a lot of significant alphas.

TABLE VI: Fixed Income Funds and Alphas

\begin{tabular}{|c|c|c|c|c|c|c|c|c|}
\hline \multicolumn{9}{|c|}{ Fund Category: Fixed Income } \\
\hline \multirow[b]{2}{*}{ Market } & \multirow[b]{2}{*}{$\begin{array}{c}\text { Risk-Free } \\
\text { Rate }\end{array}$} & \multicolumn{2}{|c|}{ Number of Active Funds } & \multicolumn{2}{|c|}{ Number of Passive Funds } & \multicolumn{3}{|c|}{ Total } \\
\hline & & $\begin{array}{c}\text { With } \\
\text { Significant } \\
\text { Alpha }\end{array}$ & $\begin{array}{c}\text { Without } \\
\text { Significant } \\
\text { Alpha }\end{array}$ & $\begin{array}{c}\text { With } \\
\text { Significant } \\
\text { Alpha }\end{array}$ & $\begin{array}{c}\text { Without } \\
\text { Significant } \\
\text { Alpha }\end{array}$ & $\begin{array}{c}\text { With } \\
\text { Significant } \\
\text { Alpha }\end{array}$ & $\begin{array}{c}\text { Without } \\
\text { Significant } \\
\text { Alpha }\end{array}$ & General \\
\hline SELIC & CDI & 147 & 1 & 191 & 3 & 338 & 4 & 342 \\
\hline SELIC & Savings & 130 & 18 & 174 & 20 & 304 & 38 & 342 \\
\hline CDI & Savings & 132 & 16 & 175 & 19 & 307 & 35 & 342 \\
\hline Category Average & CDI & 129 & 19 & 158 & 36 & 287 & 55 & 342 \\
\hline Category Average & Savings & 127 & 21 & 169 & 25 & 296 & 46 & 342 \\
\hline Category Average & SELIC & 128 & 20 & 157 & 37 & 285 & 57 & 342 \\
\hline General Average & CDI & 21 & 127 & 19 & 175 & 40 & 302 & 342 \\
\hline General Average & Savings & 132 & 16 & 181 & 13 & 313 & 29 & 342 \\
\hline General Average & SELIC & 21 & 127 & 19 & 175 & 40 & 302 & 342 \\
\hline
\end{tabular}


Finally in table VI we have the results for fixed income mutual funds. The majority of funds have significant Alphas, again we have a clear evidence of value aggregation or destruction.

\section{Management Value}

Our first conclusion, looking at the results, is that generally we have value aggregation or destruction. The exception is the exchange rate category. The next question is: managers adds or destroy value? And more, is there a difference between active and passive managers?

In order to answer these questions we analyze the mutual funds that show significant alphas. Table VII presents the results for stock mutual funds. The mean test for the active mutual funds alpha against the passive ones shows differences between them in all the cases. And with few exceptions, like in the case of IbrX use as benchmark and savings as risk-free rate and General average as benchmarks at the same time, the active managed funds add value and the passive ones destroy value. Even the passive with positive alphas destroy value. The superiority of active managed funds is clear. Table V shows the results for stock mutual funds:

TABLE VII: Stock Funds with Significant Alphas

\begin{tabular}{|c|c|c|c|c|c|c|c|c|c|}
\hline \multicolumn{10}{|c|}{ Fund Category: Stocks } \\
\hline \multirow[b]{2}{*}{ Market } & \multirow[b]{2}{*}{$\begin{array}{c}\text { Risk-Free } \\
\text { Rate }\end{array}$} & \multicolumn{3}{|c|}{ Active Funds with Significant Alphas } & \multicolumn{3}{|c|}{ Passive Funds with Significant Alphas } & \multirow[b]{2}{*}{$\begin{array}{l}\text { Mean Test } \\
(5 \%)\end{array}$} & \multirow[b]{2}{*}{$\begin{array}{l}\text { Variance } \\
\text { Test }(5 \%)\end{array}$} \\
\hline & & Mean & $\begin{array}{l}\text { Standard } \\
\text { Deviation }\end{array}$ & Number & Mean & $\begin{array}{l}\text { Standard } \\
\text { Deviation }\end{array}$ & Number & & \\
\hline IBOVESPA & CDI & 0,0046 & 0,0087 & 63 & $-0,0065$ & 0,0054 & 20 & diferentes & diferentes \\
\hline IBOVESPA & Savings & 0,0065 & 0,0081 & 66 & $-0,0064$ & 0,0052 & 20 & diferentes & diferentes \\
\hline IBOVESPA & SELIC & 0,0046 & 0,0087 & 63 & $-0,0065$ & 0,0054 & 20 & diferentes & diferentes \\
\hline $\mathrm{IBrX}$ & CDI & $-0,0066$ & 0,0076 & 73 & $-0,0125$ & 0,0047 & 30 & diferentes & diferentes \\
\hline $\mathrm{IBrX}$ & Savings & $-0,0060$ & 0,0084 & 73 & $-0,0129$ & 0,0044 & 30 & diferentes & diferentes \\
\hline $\mathrm{IBrX}$ & SELIC & $-0,0066$ & 0,0076 & 73 & $-0,0125$ & 0,0047 & 30 & diferentes & diferentes \\
\hline Category Average & CDI & 0,0021 & 0,0086 & 72 & $-0,0070$ & 0,0051 & 27 & diferentes & diferentes \\
\hline Category Average & Savings & 0,0023 & 0,0086 & 78 & $\begin{array}{l}-0,0076 \\
\end{array}$ & 0,0049 & 28 & diferentes & diferentes \\
\hline Category Average & SELIC & 0,0021 & 0,0086 & 72 & $\begin{array}{l}-0,0070 \\
\end{array}$ & 0,0051 & 27 & diferentes & diferentes \\
\hline \begin{tabular}{|l|} 
General Average \\
\end{tabular} & CDI & 0,0061 & 0,0067 & 120 & $\begin{array}{l}-0,0015 \\
\end{array}$ & 0,0062 & 25 & diferentes & iguais \\
\hline General Average & Savings & $-0,0171$ & 0,0081 & 126 & $\begin{array}{l}-0,0268 \\
\end{array}$ & 0,0054 & 34 & diferentes & diferentes \\
\hline \begin{tabular}{|l|} 
General Average \\
\end{tabular} & SELIC & 0,0062 & 0,0067 & 121 & $\begin{array}{l}-0,0011 \\
\end{array}$ & 0,0059 & 28 & diferentes & iguais \\
\hline
\end{tabular}

For hedge funds, as show in Table VIII, we conclude the same as in stock funds: active management add value and passive management destroy value. The passive ones here are the so called balanced funds, the ones that have a fixed proportion between stocks and fixed income.

TABLE VIII: Hedge Funds with Significant Alphas

\begin{tabular}{|c|c|c|c|c|c|c|c|c|c|}
\hline \multicolumn{10}{|c|}{ Fund Category: Hedge } \\
\hline \multirow[b]{2}{*}{ Market } & \multirow[b]{2}{*}{$\begin{array}{c}\text { Risk-Free } \\
\text { Rate }\end{array}$} & \multicolumn{3}{|c|}{ Active Funds with Significant Alphas } & \multicolumn{3}{|c|}{ Passive Funds with Significant Alphas } & \multirow[b]{2}{*}{$\begin{array}{c}\text { Mean Test } \\
(5 \%)\end{array}$} & \multirow[b]{2}{*}{$\begin{array}{l}\text { Variance } \\
\text { Test (5\%) }\end{array}$} \\
\hline & & Mean & $\begin{array}{l}\text { Standard } \\
\text { Deviation }\end{array}$ & Number & Mean & $\begin{array}{l}\text { Standard } \\
\text { Deviation }\end{array}$ & Number & & \\
\hline IBOVESPA & CDI & nd & nd & 0 & $-0,0045$ & 0,0025 & 3 & nd & nd \\
\hline IBOVESPA & Savings & 0,0167 & 0,0011 & 2 & 0,0045 & 0,0006 & 5 & nd & nd \\
\hline IBOVESPA & SELIC & nd & nd & 0 & $-0,0046$ & 0,0025 & 3 & nd & nd \\
\hline SELIC & CDI & 0,0024 & 0,0055 & 117 & $-0,0012$ & 0,0021 & 21 & diferentes & diferentes \\
\hline SELIC & Savings & $-0,0048$ & 0,0113 & 90 & $-0,0211$ & 0,0142 & 19 & diferentes & iguais \\
\hline Category Average & CDI & 0,0010 & 0,0038 & 46 & $-0,0027$ & 0,0025 & 18 & diferentes & diferentes \\
\hline Category Average & Savings & 0,0030 & 0,0075 & 108 & $-0,0161$ & 0,0177 & 20 & diferentes & iguais \\
\hline Category Average & SELIC & 0,0010 & 0,0038 & 44 & $-0,0026$ & 0,0025 & 18 & diferentes & diferentes \\
\hline General Average & CDI & 0,0042 & 0,0061 & 14 & $-0,0014$ & 0,0032 & 8 & diferentes & diferentes \\
\hline General Average & Savings & 0,0069 & 0,0024 & 112 & $-0,0015$ & 0,0074 & 18 & diferentes & iguais \\
\hline General Average & SELIC & 0,0037 & 0,0062 & 15 & $-0,0014$ & 0,0030 & 9 & diferentes & diferentes \\
\hline
\end{tabular}


The evidence for Fixed Income Funds is interesting. When using the same benchmark as the one used by managers, CDI or Selic, we can see that managers destroy value, in active or in passive funds. Table IX shows the results:

Table IX: Fixed Income Funds with Significant Alphas

\begin{tabular}{|c|c|c|c|c|c|c|c|c|c|}
\hline \multicolumn{8}{|c|}{ Fund Category: Fixed Income } & \multirow[b]{3}{*}{$\begin{array}{l}\text { Mean Test } \\
(5 \%)\end{array}$} & \multirow[b]{3}{*}{$\begin{array}{r}\text { Variance } \\
\text { Test (5\%) }\end{array}$} \\
\hline \multirow[b]{2}{*}{ Market } & \multirow[b]{2}{*}{$\begin{array}{c}\text { Risk-Free } \\
\text { Rate }\end{array}$} & \multicolumn{3}{|c|}{ Active Funds with Significant Alphas } & \multicolumn{3}{|c|}{ Passive Funds with Significant Alphas } & & \\
\hline & & Mean & $\begin{array}{l}\text { Standard } \\
\text { Deviation }\end{array}$ & Number & Mean & $\begin{array}{l}\text { Standard } \\
\text { Deviation }\end{array}$ & Number & & \\
\hline SELIC & CDI & $-0,0008$ & 0,0025 & 147 & $-0,0008$ & 0,0015 & 191 & iguais & diferentes \\
\hline SELIC & Savings & $-0,0044$ & 0,0039 & 130 & $-0,0030$ & 0,0018 & 174 & diferentes & diferentes \\
\hline CDI & Savings & $-0,0044$ & 0,0039 & 132 & $-0,0030$ & 0,0018 & 175 & diferentes & diferentes \\
\hline \begin{tabular}{|l|} 
Category Average \\
\end{tabular} & CDI & 0,0000 & 0,0027 & 129 & 0,0000 & 0,0018 & 158 & iguais & diferentes \\
\hline Category Average & Savings & $-0,0007$ & 0,0040 & 127 & 0,0005 & 0,0031 & 169 & diferentes & diferentes \\
\hline Category Average & SELIC & 0,0001 & 0,0027 & 128 & 0,0000 & 0,0018 & 157 & iguais & diferentes \\
\hline General Average & CDI & $-0,0054$ & 0,0034 & 21 & $-0,0047$ & 0,0016 & 19 & iguais & diferentes \\
\hline General Average & Savings & 0,0061 & 0,0015 & 132 & 0,0061 & 0,0010 & 181 & iguais & diferentes \\
\hline General Average & SELIC & $-0,0055$ & 0,0034 & 21 & $-0,0047$ & 0,0016 & 19 & iguais & diferentes \\
\hline
\end{tabular}

As a first conclusion, we can say that stocks and hedge funds add value, meanwhile fixed income ones destroy value.

\section{Jensen's Alpha Determinants}

Now we will look for the Alpha's determinants. Do to that we will run the two regressions showed in the methodology.

Table $\mathrm{X}$ shows the first regression results for stock funds:

\section{TABLE X: Stock Funds: First Regression}

\begin{tabular}{|c|c|c|c|c|c|c|c|}
\hline \multicolumn{8}{|c|}{ Fund Category: Stocks } \\
\hline \multirow[b]{2}{*}{ Market } & \multirow{2}{*}{$\begin{array}{c}\text { Risk-Free } \\
\text { Rate }\end{array}$} & \multicolumn{5}{|c|}{ Regression Coefficients } & \multirow[b]{2}{*}{$\mathbf{R 2}$} \\
\hline & & Intercept & Active (dummy) & Ln(NAV) & $\begin{array}{l}\text { National } \\
\text { (dummy) }\end{array}$ & Ln(Age in days) & \\
\hline IBOVESPA & CDI & $0,04507 * * *$ & $0,0088 * * *$ & 0,0025 **** & $-0,0003$ & $-0,0114 * * *$ & 0,625 \\
\hline IBOVESPA & Savings & $0,03993 * * *$ & $0,0104 * * *$ & $0,0025 * * *$ & 0,0011 & $-0,0109 * * *$ & 0,662 \\
\hline IBOVESPA & SELIC & $0,04506 * * *$ & $0,0088 * * *$ & 0,0025 **** & $-0,0003$ & $-0,0114 * * *$ & 0,625 \\
\hline $\mathrm{IBrX}$ & CDI & $0,03958 * * *$ & $0,0056 * * *$ & 0,0021 **** & 0,0000 & $-0,0107 * * *$ & 0,528 \\
\hline $\mathrm{IBrX}$ & Savings & $0,04444 * * *$ & $0,0062 * * *$ & 0,0024 **** & 0,0007 & $-0,0119 * * *$ & 0,549 \\
\hline $\mathrm{IBrX}$ & SELIC & $0,03954 * * *$ & 0,0056 *** & 0,0021 **** & 0,0000 & $-0,0107 * * *$ & 0,528 \\
\hline Category Average & CDI & $0,05517 * * *$ & $0,0072 * * *$ & $0,0022 * * *$ & 0,0001 & $-0,0121 * * *$ & 0,584 \\
\hline Category Average & Savings & $0,06062 * * *$ & $0,0082 * * *$ & 0,0022 **** & 0,0006 & $-0,0129 * * *$ & 0,599 \\
\hline Category Average & SELIC & $0,05515 * * *$ & $0,0072 * * *$ & $0,0022 * * *$ & 0,0001 & $-0,0121 * * *$ & 0,584 \\
\hline General Average & CDI & $0,03997 * * *$ & $0,0068 * * *$ & 0,0019 **** & 0,0002 & $-0,0091 * * *$ & 0,491 \\
\hline General Average & Savings & $0,02903 * *$ & $0,0094 * * *$ & 0,0017 *** & $0,0018 *$ & $-0,0105 * * *$ & 0,431 \\
\hline General Average & SELIC & $0,03748 * * *$ & 0,0066 *** & 0,0020 **** & 0,0002 & $-0,0089 * * *$ & 0,498 \\
\hline
\end{tabular}

$* * *=$ level of significance $<=5 \% ; * *=$ level of significance between $5 \%$ e $10 \% ; *=$ level of significance between $10 \%$ e $15 \%$

Active management, size and age are all significant variables. The evidence shows that an old, big and active fund generates biggest alphas. At the same time we can see that there is no difference between funds managed by Brazilian financial institutions and foreign ones.

When we expand the regression, adding the risk variable, we see that the other variables maintain their significance, and the new one is also significant, with a negative sign. That means that less risk generates biggest alphas. Table XI shows the results. 


\begin{tabular}{|c|c|c|c|c|c|c|c|c|}
\hline \multicolumn{9}{|c|}{ Fund Category: Stocks } \\
\hline \multirow[b]{2}{*}{ Market } & \multirow[b]{2}{*}{$\begin{array}{l}\text { Risk-Free } \\
\text { Rate }\end{array}$} & \multicolumn{6}{|c|}{ Regression Coefficients } & \multirow[b]{2}{*}{$\mathbf{R 2}$} \\
\hline & & Intercept & Active (dummy) & Ln(NAV) & $\begin{array}{l}\text { National } \\
\text { (dummy) }\end{array}$ & Ln(Age in days) & \begin{tabular}{|c|} 
Standard \\
Deviation \\
Excess Returns
\end{tabular} & \\
\hline IBOVESPA & CDI & 0,05123 **** & 0,0081 *** & $0,0024 * * *$ & $-0,0006$ & $-0,0111 * * * *$ & $-0,0767$ & 0,629 \\
\hline IBOVESPA & Savings & 0,06876 *** & 0,0076 *** & $0,0020 * * *$ & $-0,0003$ & $-0,0099 * * *$ & $-0,3195 * * *$ & 0,721 \\
\hline IBOVESPA & SELIC & 0,05116 **** & $0,0081 * * *$ & $0,0024 * * *$ & $-0,0006$ & $-0,0111$ *** & $-0,0761$ & 0,629 \\
\hline IBrX & CDI & $0,05798 * * *$ & 0,0030 **** & $0,0018 * * *$ & $\begin{array}{l}-0,0011 \\
\end{array}$ & $-0,0087 * * *$ & $-0,3348 * * *$ & 0,627 \\
\hline $\mathrm{IBrX}$ & Savings & $0,09158 * * *$ & $0,0020 * * *$ & $0,0014 * * *$ & $-0,0016 * *$ & $-0,0092 * * *$ & $-0,6021 * * *$ & 0,778 \\
\hline $\mathrm{IBrX}$ & SELIC & $0,05790 * * *$ & 0,0030 **** & $0,0018 * * *$ & $-0,0011$ & $-0,0087$ **** & $-0,3342 * * *$ & 0,626 \\
\hline \begin{tabular}{|l|} 
Category Average \\
\end{tabular} & CDI & $0,06508 * * *$ & $0,0061 * * *$ & $0,0021 * * *$ & $-0,0004$ & $-0,0115 * * *$ & $-0,1440 * *$ & 0,599 \\
\hline Category Average & Savings & 0,09356 **** & $0,0050 * * *$ & 0,0017 **** & $\begin{array}{l}-0,0009 \\
\end{array}$ & $-0,0112 * * *$ & $-0,4346 * * *$ & 0,709 \\
\hline Category Average & SELIC & 0,06501 *** & 0,0061 *** & $0,0021 * * *$ & $-0,0004$ & $-0,0115 * * *$ & $-0,1433 * *$ & 0,599 \\
\hline General Average & CDI & 0,04396 *** & 0,0065 *** & $0,0019 * * *$ & 0,0000 & $-0,0090 * * *$ & $-0,0400$ & 0,494 \\
\hline General Average & Savings & 0,07333 *** & $0,0061 * * *$ & 0,0013 *** & $-0,0001$ & $-0,0101 * * *$ & $-0,4724 * * *$ & 0,662 \\
\hline General Average & SELIC & 0,04096 **** & 0,0064 *** & 0,0020 *** & 0,0000 & $-0,0088 * * *$ & $-0,0354$ & 0,500 \\
\hline
\end{tabular}

Table XII shows the results for hedge funds. They are quite similar to the stock funds results. But with one difference: here the financial institution origin is significant. Hedge funds managed by Brazilian financial institutions generate biggest alphas.

TABLE XII: Hedge Funds: First Regression

\begin{tabular}{|c|c|c|c|c|c|c|c|}
\hline \multicolumn{8}{|c|}{ Fund Category: Hedge } \\
\hline \multirow[b]{2}{*}{ Market } & \multirow{2}{*}{$\begin{array}{c}\text { Risk-Free } \\
\text { Rate }\end{array}$} & \multicolumn{5}{|c|}{ Regression Coefficients } & \multirow[b]{2}{*}{$\mathbf{R 2}$} \\
\hline & & Intercept & Active (dummy) & Ln(NAV) & $\begin{array}{l}\text { National } \\
\text { (dummy) }\end{array}$ & Ln(Age in days) & \\
\hline IBOVESPA & CDI & nd & nd & nd & nd & nd & nd \\
\hline IBOVESPA & Savings & nd & nd & nd & nd & nd & nd \\
\hline IBOVESPA & SELIC & nd & nd & nd & nd & nd & nd \\
\hline $\begin{array}{l}\text { SELIC } \\
\end{array}$ & CDI & 0,01378 & $0,0033 * * *$ & 0,0001 & 0,0008 & $-0,0021$ & 0,075 \\
\hline \begin{tabular}{|l} 
SELIC \\
\end{tabular} & Savings & $-0,01723$ & $0,0141 * * *$ & 0,0026 *** & $0,0049 * * *$ & $-0,0064 *$ & 0,318 \\
\hline Category Average & CDI & $-0,00188$ & $0,0027 * * *$ & 0,0011 **** & $0,0021 * * *$ & $-0,0027 * *$ & 0,437 \\
\hline Category Average & Savings & $-0,02271$ & $0,0173 * * *$ & 0,0013 **** & $0,0060 * * *$ & $-0,0025$ & 0,410 \\
\hline Category Average & SELIC & $-0,00283$ & $0,0026 * * *$ & 0,0011 *** & $0,0020 * * *$ & $-0,0026 * *$ & 0,429 \\
\hline General Average & CDI & 0,00597 & $0,0040 *$ & 0,0014 **** & 0,0002 & $-0,0040$ & 0,422 \\
\hline General Average & Savings & 0,00696 & 0,0077 *** & $0,0004 * * *$ & $0,0029 * * *$ & $-0,0021 * *$ & 0,516 \\
\hline General Average & SELIC & 0,00988 & 0,0036 & 0,0015 **** & 0,0005 & $-0,0046$ & 0,402 \\
\hline
\end{tabular}

$* * *=$ level of significance $<=5 \% ; * *=$ level of significance between $5 \%$ e $10 \% ; *=$ level of significance between $10 \%$ e $15 \%$

The evidence about the role of risk in hedge funds is mixed as we can see in table XII. When savings is the risk-free rate the evidence shows that less risk generates biggest alphas. By other side, when the risk-free rate used is the CDI or Selic, more risk generates biggest alphas. Table XIII shows the results.

TABLE XIII: Hedge Funds: Second Regression

\begin{tabular}{|c|c|c|c|c|c|c|c|c|}
\hline \multicolumn{9}{|c|}{ Fund Category: Hedge } \\
\hline \multirow[b]{2}{*}{ Market } & \multirow[b]{2}{*}{$\begin{array}{c}\text { Risk-Free } \\
\text { Rate }\end{array}$} & \multicolumn{6}{|c|}{ Regression Coefficients } & \multirow[b]{2}{*}{$\mathbf{R 2}$} \\
\hline & & Intercept & Active (dummy) & Ln(NAV) & $\begin{array}{l}\text { National } \\
\text { (dummy) }\end{array}$ & Ln(Age in days) & $\begin{array}{c}\text { Standard } \\
\text { Deviation } \\
\text { Excess Returns }\end{array}$ & \\
\hline IBOVESPA & CDI & nd & nd & nd & nd & nd & nd & nd \\
\hline IBOVESPA & Savings & nd & nd & nd & nd & nd & nd & nd \\
\hline IBOVESPA & SELIC & nd & nd & nd & nd & nd & nd & nd \\
\hline SELIC & CDI & $-0,00102$ & 0,0056 *** & $0,0004 *$ & 0,0016 ** & $-0,0016$ & $0,2167 * * *$ & 0,269 \\
\hline SELIC & Savings & 0,02644 & $0,0063 * * *$ & 0,0014 *** & $0,0028 * *$ & $-0,0072 * * *$ & $-0,6677 * * *$ & 0,637 \\
\hline Category Average & CDI & $-0,00427$ & 0,0028 **** & 0,0012 **** & $0,0022 * * *$ & $-0,0027 * *$ & 0,0335 & 0,451 \\
\hline Category Average & Savings & $0,02414 * *$ & 0,0089 *** & 0,0004 & 0,0031 *** & $-0,0041$ **** & $-0,7170 * * *$ & 0,827 \\
\hline Category Average & SELIC & $-0,00640$ & 0,0028 **** & 0,0013 **** & 0,0022 **** & $-0,0026 * *$ & $0,0429 *$ & 0,450 \\
\hline General Average & CDI & $-0,00856$ & 0,0021 & 0,0024 *** & 0,0035 & $-0,0052$ & $0,1896 * * *$ & 0,609 \\
\hline General Average & Savings & $0,01655 * * *$ & 0,0057 *** & $0,0003 * *$ & 0,0022 **** & $-0,0027$ **** & $-0,1551$ *** & 0,619 \\
\hline General Average & SELIC & $-0,00520$ & 0,0018 & 0,0025 *** & 0,0032 & $-0,0057 * *$ & $0,1915 * * *$ & 0,624 \\
\hline
\end{tabular}

$* * *=$ level of significance $<=5 \% ; * *$ level of significance between $5 \%$ e $10 \% ; *=$ level of significance between $10 \%$ e $15 \%$ 
Finally we look at the fixed income funds. There is weak evidence about the role of active management in destroying value, Brazilian financial institutions reducing alphas and also the fund age in reducing alphas. More robust are the evidences relating fund size and biggest alphas. Table XIV shows the results.

TABLE XIV: Fixed Income Funds: First Regression

\begin{tabular}{|c|c|c|c|c|c|c|c|}
\hline \multicolumn{8}{|c|}{ Fund Category: Fixed Income } \\
\hline \multirow[b]{2}{*}{ Market } & \multirow{2}{*}{$\begin{array}{c}\text { Risk-Free } \\
\text { Rate }\end{array}$} & \multicolumn{5}{|c|}{ Regression Coefficients } & \multirow[b]{2}{*}{$\mathbf{R 2}$} \\
\hline & & Intercept & Active (dummy) & Ln(NAV) & $\begin{array}{l}\text { National } \\
\text { (dummy) }\end{array}$ & Ln(Age in days) & \\
\hline SELIC & CDI & $0,01025 * * *$ & 0,0002 & $0,0002 * * *$ & $-0,0004 * * *$ & $-0,0019 * * *$ & 0,164 \\
\hline SELIC & Savings & $-0,00118$ & $-0,0014$ *** & 0,0001 ** & $-0,0001$ & $-0,0006$ & 0,070 \\
\hline CDI & Savings & $-0,00099$ & $-0,0014 * * *$ & $0,0001 *$ & 0,0000 & $-0,0006$ & 0,066 \\
\hline Category Average & CDI & 0,00972 **** & 0,0002 & $0,0004 * * *$ & $-0,0001$ & $-0,0021 * * *$ & 0,212 \\
\hline Category Average & Savings & $0,01214 * *$ & $-0,0010$ *** & $-0,0001$ & $-0,0012$ *** & $-0,0011 *$ & 0,064 \\
\hline Category Average & SELIC & $0,01034 * * *$ & 0,0003 & $0,0004 * * *$ & $-0,0001$ & $-0,0021 * * *$ & 0,212 \\
\hline General Average & CDI & $-0,00974$ & $-0,0004$ & 0,0002 & $-0,0001$ & 0,0001 & 0,055 \\
\hline General Average & Savings & 0,01167 *** & 0,0000 & 0,0000 & $-0,0003 * *$ & $-0,0008 * * *$ & 0,046 \\
\hline General Average & SELIC & $-0,00979$ & $-0,0004$ & 0,0002 & $-0,0001$ & 0,0001 & 0,055 \\
\hline
\end{tabular}

Including the risk variable we see almost the same as in the previous regression, with a more strong relationship between fund age and biggest alphas. The evidence about the impact of risk in generating alphas is inconclusive, as we can see in table XV:

TABLE XV: Fixed Income Funds: Second Regression

\begin{tabular}{|c|c|c|c|c|c|c|c|c|}
\hline \multicolumn{9}{|c|}{ Fund Category: Fixed Income } \\
\hline \multirow[b]{2}{*}{ Market } & \multirow[b]{2}{*}{$\begin{array}{c}\text { Risk-Free } \\
\text { Rate }\end{array}$} & \multicolumn{6}{|c|}{ Regression Coefficients } & \multirow[b]{2}{*}{$\mathbf{R 2}$} \\
\hline & & Intercept & Active (dummy) & $\operatorname{Ln}(\mathrm{NAV})$ & $\begin{array}{l}\text { National } \\
\text { (dummy) }\end{array}$ & Ln(Age in days) & $\begin{array}{c}\text { Standard } \\
\text { Deviation } \\
\text { Excess Returns }\end{array}$ & \\
\hline SELIC & CDI & $0,00940 * * *$ & 0,0001 & $0,0002 * * *$ & $-0,0004 * * *$ & $-0,0018 * * *$ & $0,0650 *$ & 0,170 \\
\hline SELIC & Savings & 0,00647 & $-0,0006 * * *$ & $0,0001 * *$ & 0,0001 & $-0,0012$ & $-0,5207 * * *$ & 0,222 \\
\hline CDI & Savings & 0,00654 & $-0,0006 * *$ & 0,0001 & 0,0001 & $-0,0012 * * *$ & $-0,5268 * * *$ & 0,220 \\
\hline Category Average & CDI & 0,00492 & $-0,0002$ & $0,0004 * * *$ & $-0,0002$ & $-0,0016 * * *$ & $0,2987 * * *$ & 0,306 \\
\hline Category Average & Savings & 0,02208 *** & 0,0003 & $-0,0001 * *$ & $-0,0010$ **** & $-0,0020 * * *$ & $-0,9133$ **** & 0,344 \\
\hline Category Average & SELIC & $0,00551 *$ & $-0,0002$ & $0,0004 * * *$ & $-0,0002$ & $-0,0017 * * *$ & $0,3052 * * *$ & 0,309 \\
\hline General Average & CDI & $-0,01687$ & $-0,0014$ & 0,0002 & 0,0004 & 0,0009 & $0,3172 * * *$ & 0,233 \\
\hline General Average & Savings & $0,01184 * * *$ & 0,0000 & 0,0000 & $-0,0003 * *$ & $-0,0008 * * *$ & $-0,0134$ & 0,046 \\
\hline General Average & SELIC & $-0,01689$ & $-0,0014$ & 0,0002 & 0,0004 & 0,0009 & $0,3174 * * *$ & 0,233 \\
\hline
\end{tabular}

\section{Conclusions}

Our initial question was: is it better to invest in active or passive mutual funds? We do not have a simple answer. For some categories the answer is yes, for other the answer is no. For hedge funds and stock funds is clearly that active management adds value. That is, managers have a superior capacity in managing the investor's resources. In the case of fixed income the evidence is against active management. Active managers destroy value. It is important remember that fixed income mutual funds represents almost $85 \%$ of the total NAV in Brazilian mutual fund industry. For exchange rate funds there was no significant Jensen's alphas and the few ones that we found clearly indicates the value destruction. 
The analysis of alpha's determinants indicates also that they depend on the mutual fund category. For stock funds the investors have to choose active, old and big ones. And also funds with lower risk. The manager's origin has no effect in generating alphas.

Also in the hedge funds category investors must look for active, old and big ones. But here the manager's origin is important and Brazilian managers show better results. And more risky bring better results when we use the same benchmarks that are used by managers: CDI and Selic.

In the fixed income category the results are not clear. Only the relationship between size and alphas is a clear one. Big funds generate biggest alphas.

As a general conclusion we can say that there is evidence of value aggregation in active management in stock and hedge funds, but not in fixed income ones.

New research must be done with more sample segregation and also with the analysis of a broad range of alpha determinants.

\section{References:}

1. CAHART, M.M.- (1997)- On Persistence of Mutual Fund Performance - The Journal of Finance, Vol 52, No 1 , March, pp 57-82

2. CHANG, E.C.; LEWELLEN, W.G. - (1984) - "Market timing and mutual fund investment performance", Journal of Business, v.57, p.57-72.

3. GRINBLAT, M. ; TITMAN, S. - (1989) - Mutual Fund Performance: An Analysis of Quarterly Portfolio Holdings - The Journal of Business, Vol 62, nº 3, July, pp 393-416

4. HENRIKSSON, R.D. - (1984) - "Market timing and mutual fund performance: an empirical investigation", Journal of Business, v.57, p.73-96.

5. JENSEN, M.C. - (1968) - The Performance of Mutual Funds in the Period 1948-1964 - Journal of Finance $23 n^{\circ} 2$ May pp 389-416

6. KACPERCZYK, M. ; SIALM, C.; ZHENG, L. - (2005) - On the Industry Concentration of Actively Managed Equity Mutual Funds - The Journal of Finance, Vol 60, nº 4, August, pp 1983-2011

7. LHABITANT, F.S. (2001) - On Swiss Timing and Selectivity: in the Quest of Alpha - Working Paper $n^{\circ} 27$ HEC Lausanne - June

8. SHARPE, W. F. - (1991) - The Arithmetic of Active Management - Financial Analysts' Journal - Vol 47 No 1 Jan/Feb pp 7-9 
9. WERMERS, R. (2000) - Mutual Fund Performance: An Empirical Decomposition in Stock-Picking Talent, Style, Transaction Costs, and Expenses - The Journal of Finance, Vol 55, nº 4, August, pp 1655-1695

10. WHITE, H. - (1980) “A heteroscedasticity-consistent covariance matrix estimator and a direct test for heteroscedasticity", Econometrica, v.48, p. 817-838 